\title{
Electrical Accidents in the Mining Industry, 1990-1999
}

\author{
James C. Cawley, P.E., Senior Member, IEEE \\ National Institute for Occupational Safety and Health \\ Office for Mine Safety and Health Research, Pittsburgh Research Laboratory \\ 626 Cochrans Mill Road, P.O. Box 18070, \\ Pittsburgh, PA 15236, USA
}

\begin{abstract}
This National Institute for Occupational Safety and Health study was conducted to focus future research on the most significant electrical problems in the mining industry. Data from 1,926 mine electrical accidents (including 75 fatalities) that occurred between 1990 and 1999 were studied. Coal and metalnonmetal operator- and contractor-reported data are presented. All data used in this analysis were MSHA closeout data, except 1999, which were preliminary data.
\end{abstract}

Electricity was the 4th leading cause of death reported in mining despite being the 14th leading cause of injuries. During the 1990s, 1 of every 272 mining accidents resulted in a fatality. In comparison, 1 of every 26 mine electrical accidents was fatal. Burns were the leading Nature of Injury in electrical accidents, but were rarely fatal. Electrical shock caused 70 of the 75 electrical fatalities reported. About one-half of mine electrical accidents and fatalities were sustained during electrical maintenance. Small mines experience a disproportionately high number of electrical accidents and fatalities based on total average employment. The injury severity for victims of nonfatal mine electrical injuries does not increase with age in victims 50 years and older, unlike many other types of occupational accidents. High-reaching mobile equipment is involved in about $\mathbf{2 0 \%}$ of mine electrical fatalities, indicating that overhead power line hazards need to be addressed. Electrical accident narratives containing the six most frequently mentioned keywords were isolated for further analysis. 1,321 narratives containing the keywords "breaker", "cable", "battery", "ground", "energized" and "meter" were analyzed to more accurately determine causal factors. Technical suggestions for mitigating electrical hazards are proposed.

\section{INTRODUCTION}

This study was conducted to focus future research on the most significant electrical problems in the mining industry. In addition, it formed the first phase of a larger effort to identify electrical hazards common to both mining and other industries.

The Mine Safety and Health Administration (MSHA) is empowered by statute to collect detailed information on accidents, injuries and illnesses that occur in the mining industry. MSHA also collects information about mines, employment and production. The accident data are compiled from information on the MSHA Form 7000-1, Mine Accident, Injury, and Illness Report. Data on mines (active, inactive and abandoned), employment and production (for coal mines) are reported on the MSHA Form 7000-2, Quarterly Mine Employment and Coal Production Report. Mine operators are required to report accidents, injuries, illnesses and certain other "reportable accidents" " that occur to bothemployees and nonemployees on mine property. This statutory reporting requirement has allowed MSHA to amass one of the best publicly accessible occupational injury databases available in the U.S. The Mine Accident and Injury (AI) and Mine Address and Employment (AE) databases consolidate several MSHA raw databases to provide, for example, ready association of accident narratives with other accident information. Information for this paper was compiled from the $\mathrm{AI}$ and $\mathrm{AE}$ databases covering the period from 1990-1999. All data used in this report were MSHA closeout data except 1999, which used preliminary data available through the fourth quarter of 1999.

\section{BACKGROUND}

Between 1990 and 1999 mining operators and contractors reported 260,510 accidents, injuries and illnesses from all causes, including 959 fatalities. Mines reported 1,926 electrical accidents, including 75 fatalities. Electricity was the 4 th leading cause of death in mining despite ranking 14th overall as an accident cause. Nonfatal mining electrical accidents were responsible for 31,370 lost work days (LWDs).

The coal industry is made up of two Standard Industrial Classifications (SICs), anthracite coal and bituminous coal. Anthracite (hard coal) is a small segment of the coal industry in terms of both production and total accidents. During the study period, coal operators reported 129,553 accidents, injuries and illnesses from all causes, including 379 fatalities. The number of active coal mines (those characterized by reporting 1 or more hours of work in a given year) fell each year from 4,320 in 1990 to 2,301 in 1999 and coal operator employment decreased $43 \%$, from 145,887 to 82,907 . Coal contractor employment, however, increased by $40 \%$, from 21,938 in 1990, to 30,812 in 1999, peaking at 32,201 in 1997. LWD accidents from all causes reported by coal operators showed a decline of $73 \%$, from 11,381 cases in 1990 to 3,055 in 1999.

The metal-nonmetal (MNM) sector is made up of 86 different SICs representing a wide range of commodities,

\footnotetext{
${ }^{1}$ For a more precise definition of "reportable accidents" see [1].
} 


\begin{tabular}{|c|c|}
\hline \multicolumn{2}{|c|}{$\begin{array}{c}\text { TABLE } 1 \\
\text { Percent OF Mining Accidents That RESUlted IN A FATAlity, 1990-1999 }\end{array}$} \\
\hline Category & Percent Fatal \\
\hline Falling/rolling/sliding rock or material of any kind & $10.69 \%$ \\
\hline Explosives and breaking agents & $7.92 \%$ \\
\hline Electrical & $3.89 \%$ \\
\hline Fall of face/rib/side/highwall & $2.39 \%$ \\
\hline Exploding vessels under pressure & $2.03 \%$ \\
\hline Ignition/explosion of gas or dust & $1.85 \%$ \\
\hline Haulage (powered) & $1.42 \%$ \\
\hline Fall of roof or back & $0.54 \%$ \\
\hline Fire (not electrical or explosion) & $0.53 \%$ \\
\hline Machinery & $0.44 \%$ \\
\hline Not Elsewhere Classified and not an occ. illness & $0.44 \%$ \\
\hline Inundation & $0.37 \%$ \\
\hline Slip or fall of person & $0.15 \%$ \\
\hline Hoisting & $0.13 \%$ \\
\hline Hand tools & $0.05 \%$ \\
\hline \multirow[b]{2}{*}{ Average percent fatal from all causes } & $0.01 \%$ \\
\hline & $0.37 \%$ \\
\hline \multicolumn{2}{|c|}{$\begin{array}{l}\text { Notes: 1. Aggregated data for all mining SICs. } \\
\text { 2. Categories shown produced lor more fatalities per } 10,000 \\
\text { accidents. }\end{array}$} \\
\hline
\end{tabular}

mining methods and machines. From 1990 to 1999 MNM operators reported 115,350 accidents, injuries and illnesses from all causes, including 373 fatalities. The number of active MNM mines fell from 11,838 in 1990 to a low of 10,843 in 1996. It then increased each year to 11,821 in 1999. MNM operator employment declined by only $7 \%$, from 207,515 in 1990 , to 192,907 in 1999 . MNM contractor employment, however, increased by $69 \%$, from 26,460 in 1990 to 44,793 in 1999. LWD accidents from all causes reported by MNM operators declined by $64 \%$, from 7,129 cases in 1990 to 2,544 in 1999.

Certain types of mining accidents occur infrequently but cause a high number of fatalities per accident. Table 1 shows types of accidents ranked by the percent that resulted in a fatality. Mine electrical accidents are fatal in disproportion to their frequency. They rank 3 rd in the overall ratio of total accidents to total fatalities. Many high frequency accident types (i.e., handling materials, slips and falls, etc.) have a low likelihood of resulting in a fatality.

Table 2 shows the accident-to-fatality ratio for each category of employer in the mining industry. Both coal and MNM contractors reported a higher ratio of electrical fatalities to electrical accidents than did their respective operators.

\section{Mine Electrical InJuRy Data}

TABLE 2

Ratio of Mining Accidents to Fatalities From All Causes and From Electrical Causes, 1990-1999

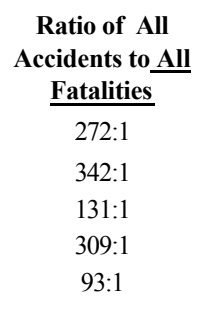

Ratio of Electrical Accidents to Electrical Fatalities $26: 1$

All Mining Coal Operators Coal Contractors MNM Operators MNM Contractors 93:1 


\begin{tabular}{|lc|}
\hline \multicolumn{2}{|c|}{ TABLE 3 } \\
\multicolumn{1}{|c|}{ DEGREE OF INJURY FOR MINE ELECTRICAL ACCIDENTS, } & 1990-1999 \\
Degree of Injury & Total Cases \\
Fatality & 75 \\
Permanent disability (partial or total) & 9 \\
Days away from work only & 1,109 \\
Days away and restricted activity & 80 \\
Days of restricted activity only & 105 \\
Injury without death, days away, or restricted activity & 441 \\
Occupational illness & 0 \\
Fatal/nonfatal injury due to natural causes & 0 \\
Fatal/nonfatal injury - nonemployee on mine property & 5 \\
NEC (first aid only, nonchargeable death/ disability & 14 \\
No injury & $\underline{88}$ \\
& Total \\
\end{tabular}

resulting from an accident. Table 5 shows that burns of all types are the most common form of electrical injury. These burns, which account for $65 \%$ of nonfatal electrical injuries, are the cause of only $7 \%$ of electrical fatalities. Electrical shock causes $24 \%$ of electrical injuries but $93 \%$ of electrical fatalities.

Table 6 shows nonfatal injury severity based on the Nature of Injury for LWD electrical accidents. Nonfatal Injury severity is measured as the average number of LWDs incurred per LWD injury. Electrical shock, the leading cause of electrical fatalities, ranks 9th in nonfatal injury severity. Radiation burns from electrical arcing, the leading cause of nonfatal electrical injury, ranks 13 th.

\section{E. Regular Job Titles of Electrical Accident Victims}

Regular Job Titles reporting 10 or more electrical injuries during the 1990s and their associated fatalities are shown in Table 7. Overall, $84 \%$ of electrical injuries and $89 \%$ of electrical fatalities reported by Regular Job Title are shown in the table.

\section{F. Work Activities Resulting in Electrical Accidents}

Table 8 shows the WorkActivity that was being performed when an electrical accident occurred. Each Work Activity that recorded 10 or more electrical injuries is shown in the table. The activities shown account for $89 \%$ of electrical injuries and $85 \%$ of electrical fatalities. Maintenance/repair (electrical) was the most hazardous electrical Work Activity, accounting for $50 \%$ of electrical accidents and $48 \%$ of electrical fatalities reported by Work Activity. Maintenance/repair (machinery - not electrical) was the second most hazardous electrical Work Activity accounting for $13 \%$ of electrical accidents and $7 \%$ of electrical fatalities.

\begin{tabular}{|c|c|c|c|}
\hline \multicolumn{4}{|c|}{$\begin{array}{c}\text { TABLE } 4 \\
\text { Mine ELECTRICAL ACCIDENTS By SIC, } 1990-1999\end{array}$} \\
\hline$\underline{\text { SIC }}$ & Sector Description & Injuries & Fatalities \\
\hline 12110 & Coal, Bituminous & 982 & 33 \\
\hline 14220 & Limestone (crushed and broken) & 240 & 8 \\
\hline 14410 & Sand and gravel & 202 & 7 \\
\hline 10210 & Copper ore & 64 & 6 \\
\hline 32410 & Cement & 54 & 1 \\
\hline 10410 & Gold (lode and placer) & 50 & 3 \\
\hline 14550 & Clay (common) & 43 & 2 \\
\hline 14230 & Granite (crushed and broken) & 34 & 2 \\
\hline 14750 & Phosphate rock & 28 & 2 \\
\hline 14294 & Traprock (crushed and broken) & 22 & 2 \\
\hline 14742 & Potash & 20 & 2 \\
\hline 10310 & Lead and/or zinc ore & 17 & 1 \\
\hline 14290 & Stone (crushed and broken, NEC) & 17 & 2 \\
\hline 10110 & Iron Ore & 15 & 0 \\
\hline 28191 & Alumina (Mill) & 13 & 0 \\
\hline 14743 & Trona & 13 & 1 \\
\hline 11110 & Coal, Anthracite & $\underline{12}$ & $\underline{3}$ \\
\hline & & 1,826 & 75 \\
\hline \multicolumn{4}{|c|}{$\begin{array}{l}\text { Notes: 1. Injury totals include fatalities. } \\
\text { 2. } 100 \text { additional injuries from SIC's that reported } 10 \text { or fewer } \\
\text { electrical injuries are not shown. } \\
\text { 3. All fatalities are shown. }\end{array}$} \\
\hline
\end{tabular}

\section{G. Machines Involved in Electrical Accidents}

Table 9 shows the Machine Types involved in mine electrical accidents and fatalities. Machine Type was reported for 846 electrical accidents (44\% of all mine electrical accidents) and 39 electrical fatalities (52\% of all mine electrical fatalities). $92 \%$ of electrical accidents and $95 \%$ of the electrical fatalities that reported Machine Type are shown in Table 9. Unfortunately, for analysis purposes, the leading Machine Type reported was the Machine NEC (Not Elsewhere Classified) category accounting for $12 \%$ of the electrical injuries and $10 \%$ of the fatalities reporting Machine Type. Second most reported was the Continuous miner, tunnel borer, DOSCO category with $6 \%$ of the electrical injuries and $8 \%$ of the fatalities.

Of the 39 fatalities reporting Machine Type, 13 involved the category Crane, Derrick, Cherry picker, Boom hoist, etc., representing $33 \%$ of the total in this category and $17 \%$ of all mine electrical fatalities. However, this category reported only $5 \%$ of total mine electrical injuries reporting Machine Type. 


\begin{tabular}{|c|c|c|}
\hline \multicolumn{3}{|c|}{$\begin{array}{c}\text { TABLE } 5 \\
\text { NAtURE OF INJURY RESUlting FrOM Mine ElectricAl ACCIDENTS, } \\
1990-1999\end{array}$} \\
\hline$\underline{\text { Nature of Injury }}$ & $\underline{\text { Injuries }}$ & $\underline{\text { Fatalities }}$ \\
\hline $\begin{array}{l}\text { Radiation effects (burn from electrical arc - not } \\
\text { contact) }\end{array}$ & 770 & 3 \\
\hline Electric shock, electrocution & 447 & 70 \\
\hline Burn (electrical) & 164 & 0 \\
\hline Burn or scald (heat - not radiation) & 128 & 2 \\
\hline Burn (chemical) & 126 & 0 \\
\hline Multiple injuries & 45 & 0 \\
\hline $\begin{array}{l}\text { Sprains/strains/ruptured disc/whiplash/torn knee } \\
\text { cartilage }\end{array}$ & 32 & 0 \\
\hline Cut/laceration/puncture/infection & 28 & 0 \\
\hline $\begin{array}{l}\text { Asphyxia/strangulation/drowning/smoke } \\
\text { inhalation/suffocation }\end{array}$ & 17 & 0 \\
\hline Fracture, chip & 16 & 0 \\
\hline Contusion, bruise & 11 & 0 \\
\hline Scratches, abrasions (superficial wounds) & 9 & 0 \\
\hline NEC & 9 & 0 \\
\hline Amputation or enucleation & 7 & 0 \\
\hline Poisoning, systemic & 6 & 0 \\
\hline Dust or other particles in eyes & 3 & 0 \\
\hline Concussion - brain, cerebral & 2 & 0 \\
\hline Dislocation & 2 & 0 \\
\hline Hearing loss or impairment (industrial) & 1 & 0 \\
\hline Hernia, rupture & 1 & 0 \\
\hline Radiation effects, not elsewhere classified & 1 & 0 \\
\hline Radiation effects (sunburn) & 1 & 0 \\
\hline Total & 1,826 & 75 \\
\hline
\end{tabular}

Overhead power lines are a major causal factor in fatal mine electrical accidents involving cranes and other highreaching, mobile mining equipment. Mine electrical accidents involving overhead power lines are a disproportionately fatal accident category within the overall electrical accident category which, in itself, is disproportionately fatal.

Electrical accidents reporting Pumps as the Machine Type also showed a disproportionate number of fatalities when compared with the number of injuries. Pumps are involved in $8 \%$ of electrical fatalities but only $2 \%$ of electrical injuries that reported a Machine Type.

\section{H. Accident Severity Versus Age of the Victim}

A Bureau of Labor Statistics (BLS) study of nonfatal industrial injury severity showed that increasing age is highly correlated to increasing injury severity (average LWDs per LWD accident). [2] Fotta analyzed this trend for the mining industry and found similar results. [3] While true for accidents in general, not all accident categories follow this trend.
TABLE 6

ELECTRICAL InJURY SEVERITy By NATURE OF INJURY, 1990-1999

$\quad$ Nature of Injury
Amputation or enucleation
Fracture, chip
Dislocation
Asphyxia/ strangulation/ drowning/
smoke inhalation/ suffocation
Sprains/strains/ruptured
disc/whiplash/torn knee cartilage
Burn or scald (heat - not radiation)
Burn (electrical)
NEC
Electric shock, electrocution
Scratches, abrasions (superficial
wounds)
Concussion - brain, cerebral
Multiple injuries
Radiation effects (burn from electrical arc
- not contact)
Hernia, rupture
Contusion, bruise
Burn (chemical)
Cut/laceration/puncture/infection
Dust or other particles in eyes
Poisoning, systemic
Hearing loss or impairment (industrial)
Radiation effects (sunburn)
Radiation effects, not elsewhere
classified

\begin{tabular}{|c|c|c|}
\hline $\begin{array}{l}\text { Average } \\
\text { LWD per } \\
\text { LWD Injury }\end{array}$ & $\begin{array}{l}\text { No. of } \\
\text { Cases }\end{array}$ & $\begin{array}{c}\text { Total } \\
\text { LWDs }\end{array}$ \\
\hline 179 & 7 & 1,250 \\
\hline 86 & 13 & 1,122 \\
\hline 44 & 2 & 87 \\
\hline 43 & 10 & 428 \\
\hline 35 & 23 & 814 \\
\hline 34 & 87 & 2,984 \\
\hline 34 & 107 & 3,600 \\
\hline 31 & 3 & 92 \\
\hline 31 & 263 & 8,054 \\
\hline 25 & 6 & 152 \\
\hline 23 & 1 & 23 \\
\hline 21 & 32 & 677 \\
\hline 21 & 512 & 10,740 \\
\hline 16 & 1 & 16 \\
\hline 11 & 8 & 87 \\
\hline 10 & 74 & 745 \\
\hline 8 & 7 & 57 \\
\hline 5 & 1 & 5 \\
\hline 4 & 5 & 20 \\
\hline 3 & 1 & 3 \\
\hline 2 & 1 & 2 \\
\hline 1 & 1 & 1 \\
\hline 26.6 & & \\
\hline
\end{tabular}

Average nonfatal injury severity for mine electrical injuries peaks at $21 \mathrm{LWDs}$ per LWD injury for the 40-49 age group and decreases to 20 and 13 LWDs per LWD injury for the 50-59 age group and $60+$ age groups, respectively. Workers less than 20 years old average about 19 LWDs per LWD injury.

Nonfatal injury categories that dominate the LWD mining injury total include slips and falls, handling materials, etc. These accidents produce injury types (back injuries, strains, sprains, contusions, etc.) that may take longer to heal with increasing age. Electrical injuries, however, produce burns, electrical shocks, nerve and muscular damage to body systems and multiple body parts, where recovery time may be less sensitive to the victim's age.

\section{Electrical AcCident NarRative ANalysis}

\section{A. General Observation Regarding Accident Narratives}

MSHA requires a descriptive narrative to be filed for each 


\begin{tabular}{|c|c|c|c|}
\hline \multicolumn{4}{|c|}{$\begin{array}{c}\text { TABLE } 7 \\
\text { Regular Job TitLe OF MinING EleCtrical ACCidENT Victims, } \\
\text { 1990-1999 }\end{array}$} \\
\hline MSHA & $\underline{\text { Regular Job Title }}$ & $\underline{\text { Injuries }}$ & $\underline{\text { Fatalities }}$ \\
\hline \multicolumn{4}{|l|}{ Code } \\
\hline 302 & Electrician (surface) & 287 & 14 \\
\hline 304 & Mechanic/repairman (surface) & 193 & 4 \\
\hline 102 & Electrician (off section) & 126 & 6 \\
\hline 374 & Cleaning plant/media/ crusher/ & 118 & 4 \\
\hline 104 & Mechanic/repairman (off section) & 95 & 1 \\
\hline 316 & Laborer/utility man/pumper (surface) & 87 & 3 \\
\hline 116 & Laborer/muck machine operator/pipe & 58 & 2 \\
\hline 50 & Shuttle car operator/ram car (on & 53 & 2 \\
\hline 449 & Mine foreman/mine manager/owner & 47 & 2 \\
\hline 2 & Electrician (on section/face) & 46 & 2 \\
\hline 494 & Prep plant foreman/mill foreman & 46 & 3 \\
\hline 376 & Truck driver (surface) & 42 & 3 \\
\hline 46 & Roof bolter/rock bolter (on & 40 & 0 \\
\hline 149 & Labor foreman/bullgang foreman (off & 38 & 1 \\
\hline 418 & Maintenance foreman (supv/staff) & 34 & 3 \\
\hline 368 & Bulldozer operator/tractor/ heavy & 33 & 1 \\
\hline 382 & Highlift/ front end loader operator & 33 & 0 \\
\hline 481 & Superintendent (supv/staff) & 33 & 2 \\
\hline 489 & Outside foreman (supv/staff) & 33 & 3 \\
\hline 319 & Welder (surface) & 25 & 2 \\
\hline 4 & Mechanic/repairman (on section/face) & 24 & 3 \\
\hline 269 & Motorman/swamper/ switchman (UG & 21 & 0 \\
\hline 36 & Continuous miner operator/ mole (on & 18 & 0 \\
\hline 101 & Belt/conveyor man (off section) & 18 & 0 \\
\hline 402 & Master electrician (supv/staff) & 18 & 3 \\
\hline 456 & Engineer - EE/ ventilation/ mining & 15 & 1 \\
\hline 318 & Oiler/greaser (surface) & 12 & 0 \\
\hline 54 & Scoop car/unitrac operator (on & 11 & 1 \\
\hline 301 & Belt/conveyor man (surface) & 11 & 0 \\
\hline 372 & Barge/boat/dredge attendant (surface) & 11 & 1 \\
\hline & Total & 1,626 & 67 \\
\hline \multicolumn{4}{|c|}{$\begin{array}{l}\text { Notes: 1. Regular Job Title shown for each RJT reporting } 10 \\
\text { or more electrical accidents during the period. } \\
\text { 2. } 71 \text { fatalities reported Regular Job Title. } \\
\text { 3. } 1,801 \text { accidents reported Regular Job Title } \\
\text { 4. Injury totals include fatalities }\end{array}$} \\
\hline
\end{tabular}

mining accident, injury, or illness reported. These narratives varywidelyin their information content, grammar and spelling. Some are so brief as to be unusable for analyzing an accident situation while others contain a significant amount of information. In addition, MSHA subject matter experts document each fatality with a more detailed fatality report. Whencompleted, these fatality reports are publically available on the MSHA website (http://www.msha.gov).

Capelli-Schellpfeffer recommended integrating accident narrative s with statistical information to increase the reliability of electrical accident causal analyses. [4] That approach was
TABLE 8

Work Activity Being Performed When an Electrical Accident OCCURRED, 1990-1999

\begin{tabular}{|c|c|c|}
\hline Work Activity & $\underline{\text { Injuries }}$ & $\underline{\text { Fatalities }}$ \\
\hline Maintenance/repair (electrical) & 907 & 35 \\
\hline Maintenance/repair (machinery - not electrical) & 240 & 5 \\
\hline $\begin{array}{l}\text { Handling supplies/material (not timber) - } \\
\text { load/unload }\end{array}$ & 133 & 4 \\
\hline Inspect equipment (not maintenance/repair) & 104 & 3 \\
\hline Move power cable (includes reeling) & 82 & 3 \\
\hline Hand tools (not powered) & 30 & 2 \\
\hline Welding and cutting & 28 & 0 \\
\hline Escaping a hazard & 25 & 0 \\
\hline Rerail equipment (includes replace trolley pole) & 20 & 1 \\
\hline Operate surface equipment nec & 19 & 5 \\
\hline Observe operations & 17 & 1 \\
\hline Walking/running & 15 & 0 \\
\hline $\begin{array}{l}\text { Move equipment (fans/pumps, not operating } \\
\text { machinery) }\end{array}$ & 14 & 1 \\
\hline Idle (lunch, coffee break, etc.) & 13 & 0 \\
\hline Operate locomotive (air trammer) & 13 & 0 \\
\hline Operate mill equipment & 13 & 0 \\
\hline Hand tools (powered) & 12 & 2 \\
\hline Get on/off equipment, machines, etc. & 11 & 2 \\
\hline Operate continuous miner & 10 & 0 \\
\hline Total & 1,706 & 64 \\
\hline \multicolumn{3}{|c|}{$\begin{array}{l}\text { Notes: 1. Work Activities shown reported } 10 \text { or more injuries during } \\
\text { the reporting period. } \\
\text { 2. } 73 \text { fatalities reported Work Activity. } \\
\text { 3. } 1,831 \text { accidents reported Work Activity } \\
\text { 4. Injury totals include fatalities. }\end{array}$} \\
\hline
\end{tabular}

adopted for this analysis. Automated keyword searches of the MSHA database narrative information proved of significant value in the identification of causal factors in the narratives examined. Although some undercounting may occur, computerized text string searches can help investigators with subject matter familiarity rapidly key in on important problem areas. Care must be exercised to ensure that keywords are used only within their relevant contexts. Therefore, reading and manual classification of narratives selected by keywords is imperative. The narratives from 1,926 electrical accidents (including 75 fatal accidents) were examinedand the frequency of in-context relevant word usage was determined.

\section{B. Information from Selected Electrical Accident Narratives}

Several factors rapidly emerged from the analysis of accident narratives. Circuit voltage was mentioned in only 279 of 1,926 narratives. In addition, the keywords "breaker(s)" (313 of 1,926 narratives), "cable(s)" (309 accidents), "batter(y)(ies)" (242 accidents), "energize(d)" (i.e., working live) (163 accidents), "grounds/grounding" (204 accidents), 


\begin{tabular}{|c|c|c|c|}
\hline \multicolumn{4}{|c|}{$\begin{array}{c}\text { TABLE } 9 \\
\text { MACHINE TyPES INVOLVED IN Mine ElECTRICAL ACCIDENTS, 1990-1999 }\end{array}$} \\
\hline \multicolumn{2}{|c|}{ Machine } & Injuries & $\underline{\text { Fatalities }}$ \\
\hline \multicolumn{2}{|c|}{ Machine, NEC (Not Elsewhere Classified) } & 99 & 4 \\
\hline \multicolumn{2}{|c|}{ Continuous miner, tunnel borer, DOSCO } & 53 & 3 \\
\hline \multicolumn{2}{|c|}{$\begin{array}{l}\text { Mancar, mantrip, personnel carrier, portabus, } \\
\text { jeep, jitney }\end{array}$} & 49 & 1 \\
\hline \multicolumn{2}{|c|}{ Front-end loader, payloader, highlift, etc. } & 47 & 0 \\
\hline \multicolumn{2}{|c|}{ Hand tools (not powered) - wrench, jacks, etc. } & 46 & 1 \\
\hline \multicolumn{2}{|c|}{ Welding machine, bonder, torch } & 45 & 1 \\
\hline \multicolumn{2}{|c|}{ Shuttle car - buggy, torkar, ram car } & 43 & 2 \\
\hline \multicolumn{2}{|c|}{ Crane, derrick, cherry picker, boom hoist, etc. } & 42 & 13 \\
\hline \multicolumn{2}{|c|}{$\begin{array}{l}\text { Ore haulage trucks - off highway and } \\
\text { underground }\end{array}$} & 38 & 2 \\
\hline \multicolumn{2}{|c|}{$\begin{array}{l}\text { Conveyor, belt feeder, mobile bridge carrier, } \\
\text { ROSCO }\end{array}$} & 34 & 2 \\
\hline \multicolumn{2}{|c|}{ Locomotive, rail-mounted, lorry car } & 34 & 0 \\
\hline \multicolumn{2}{|c|}{ Rock or roof bolting machine } & 34 & 0 \\
\hline \multicolumn{2}{|c|}{ Crusher, breaker, mills (ball and rod) } & 31 & 0 \\
\hline \multicolumn{2}{|c|}{ Shovel or dragline (mining and stripping) } & 29 & 2 \\
\hline \multicolumn{2}{|c|}{$\begin{array}{l}\text { Load-haul-dump/ scoop tram/ CAVO/ } \\
\text { transloader/ ram car }\end{array}$} & 28 & 0 \\
\hline \multicolumn{2}{|c|}{ Bulldozer, dozer, crawler tractor, etc. } & 19 & 0 \\
\hline \multicolumn{2}{|c|}{$\begin{array}{l}\text { Trucks - pickup/dump/water/service (not ore } \\
\text { haulage) }\end{array}$} & 19 & 0 \\
\hline \multicolumn{2}{|c|}{ Drills (electric/hydraulic/coal - not impact drills) } & 18 & 0 \\
\hline \multicolumn{2}{|l|}{ Fan } & 16 & 0 \\
\hline \multicolumn{2}{|l|}{ Pump } & 16 & 3 \\
\hline \multicolumn{2}{|c|}{ Milling machinery, nec } & 15 & 1 \\
\hline \multicolumn{2}{|c|}{ Hand tools (powered) - drill, impact wrench, etc. } & 14 & 2 \\
\hline \multicolumn{2}{|c|}{ Drill (carriage-mounted) on track/rail/rubber tired } & 10 & 0 \\
\hline & Total & 779 & 37 \\
\hline Notes: & $\begin{array}{l}\text { 1. Machine Types shown reported } 10 \mathrm{c} \\
\text { during the reporting period. } \\
\text { 2. } 39 \text { fatalities reported Machine Type. } \\
\text { 3. } 846 \text { accidents reported Machine Typ } \\
\text { 4. Injury totals include fatalities. }\end{array}$ & r more inju & \\
\hline
\end{tabular}

and electrical "meter(s)" (90 accidents) were involvedinabout two-thirds of mine electrical accidents. A total of 1,321 narratives containing these six keywords were analyzed and their causal factors determined.

1. Circuit voltage: The circuit voltage is specifically mentioned in only 279 (14\%) of all mine electrical accident narratives, limiting its usefulness in determining possible accident mitigation strategies. $57 \%$ of the 243 alternating current accidents occurred at or below $600 \mathrm{Vac}, 3 \%$ between 601 and $1000 \mathrm{Vac}$ and $40 \%$ at more than $1000 \mathrm{Vac}$. Accidents involving DC circuits mention specific voltages in only 36 cases, covering the range from common battery voltages to trolley circuit voltages $(6 \mathrm{Vdc}$ to $750 \mathrm{Vdc})$. The reliability of voltage data in accident narratives could be improved by always reporting the nominal circuit voltage of an electrical accident and by specifying whether the accident occurred via phase-tophase or phase-to-ground contact.

2. Circuit breakers: 313 circuit "breaker" accidents were grouped into 8 causal categories:

- electrical maintenance or repair working live (either intentionally or unintentionally) - 125 cases;

- operating/resetting a circuit breaker - 108 cases;

- equipment failure - 22 cases;

- shocked/burned while plugging/unplugging connectors 15 cases;

- working in proximity to a live circuit - 15 cases;

- energized/de-energize the wrong circuit breaker - 14 cases;

- unknown cause - 8 cases, and;

- misclassified - 6 cases.

3. Cables: 309 cable accidents were grouped into 10 causal categories:

- handling or moving live cable, plugs, or running over live cable - 86 cases;

- jumper cables and batteries - 65 cases;

- electrical maintenance or repair working live (either intentionally or unintentionally) and plugging/unplugging live connectors, - 59 cases;

- shocked or burned unexpectedly - 31 cases;

- equipment failure (cause unknown) - 27 cases;

- power-off electrical repair accident (energizing cable after repair, working in proximity to live circuits) - 12 cases;

- touching bad splices - 12 cases;

- contact with overhead power lines - 11 cases;

- unclassifiable - 4 cases, and;

- misclassified accidents - 2 cases.

Only 12 accidents mentioned that a worker contacted a bad cable splice while 86 accidents involved workers handling cables containing previously unknown cuts and abrasions that apparently exposed live conductors.

4. Batteries: 242 battery accidents were grouped into 8 causal categories:

- battery exploded during or immediately after maintenance or repair - 110 cases;

- while using jumper cables, battery exploded - 46 cases;

- battery exploded while charging battery or plugging or unplugging charger cable - 28 cases;

- other cables arced or exploded during maintenance - 15 cases;

- battery exploded spontaneously - 15 cases;

- jumper cables arced or exploded during use - 5 cases;

- other - 21 cases, and;

- misclassified - 2 cases. 
5. Grounding: Of the 204 accidents containing the text string "ground.", only 129 represented cases that were not either double-counted from another keyword category or truly represented a grounding problem. These 129 grounding accidents were grouped into 9 causal categories:

- failure to de-energize equipment (intentionally or unintentionally) prior to grounding work - 47 cases;

- grounding system, component, or insulation defects in stationary equipment - 29 cases;

- using grounding test equipment, meters, leads - 13 cases;

- grounding defects in mobile equipment - 13 cases;

- ground conductor defects - 12 cases;

- grounding defects in portable cords - 3 cases;

- unknown causes - 8 cases, and;

- welding - 4 cases.

6. Working on energized circuits: 163 accidents occurred while working on energized electrical circuits. Injuries resulting from working on energized electrical circuits were grouped into 9 causal categories:

- knowingly failed to de-energize the circuit before beginning work - 78 cases;

- equipment failure (includes cut and abraded cables) - 37 cases;

- de-energized the wrong circuit or did not de-energize adjacent circuits - 16 cases;

- working under or near energized trolley line - 9 cases;

- using improper tools/equipment/test leads or improper use of same - 8 cases;

- circuit reenergized by another person during work - 6 cases;

- working under or near energized power line - 4 cases;

- improper cable repair (leads reversed) - 1 case, and;

- unknown cause - 4 cases.

7. Using meters and test leads for troubleshooting: 90 accident narratives mentioned that the victim was using a meter to troubleshoot an electrical circuit. Injuries resulting from using meters on energized electrical circuits were grouped into 8 causal categories:

- meter exploded (cause unspecified) - 30 cases;

- test leads/probes shorted, arced - 21 cases;

- meter used on wrong function (e.g., measured volts on ohms scale) - 13 cases;

- meter of wrong voltage used (e.g., used a $1000 \mathrm{~V}$ meter on a $4160 \mathrm{~V}$ circuit) - 10 cases;

- dropped / misused meter - 8 cases;

- victim wearing metal jewelry - 1 case;

- unknown cause - 4 cases, and;

- misclassified - 3 cases.

\section{IMPLICATIONS FoR MINE ELECTRICAL RESEARCH}

Rossignol points out that while training solutions are often suggested for electrical hazards, interventionefforts must shift toward engineering control solutions "to reduce the hazard at its source". [5] This is practical in many situations. Simple, cost effective engineering control solutions exist to reduce fatalities and mitigate severity of nonfatal electrical injuries. Manuele notes that $60 \%$ of identified barriers to safe work behaviors arise from shortcomings in facilities and equipment and 13\% from management systems. [6] "That suggests," he contends, "that the greatest risk reduction will come from attention to those two subjects". While suitable kinds and levels of training cannot be overlooked, over dependance on training at the expense of engineering control interventions, where appropriate, is a serious error.

Safety strategies common to many electrical accident prevention programs include:

1. working live only as a last resort;

2. training in the use of proper lockout-tagout procedures;

3. training in the use of appropriate PPE, including:

a. UV limiting eye or full-face protection;

b. lightweight, fire-retardant work clothes for electrical maintenance personnel and arc protection suits where needed, and;

c. the use of dry electrical gloves, insulating blankets, and other situation specific PPE as needed.

\section{A. Mitigating the Frequency and Severity of Flash Burn Injuries}

"Radiation effects (burn from electrical arc)" type injuries caused $40 \%$ of all mining electrical injuries and accounted for $34 \%$ of electrical LWDs. Such injuries are largely a consequence of working live and account for a high percentage of electrical eye and hand injuries. Only 3 fatalities were directly attributed to this Nature of Injury.

Possible mitigations for electrical arc burninjuries include limiting the available arc blast energy, therefore injury severity, by using current limiting circuit protection, eliminating or reducing intentional time delays in protective devices and using high resistance grounding where practical.

\section{B. Mitigating the Frequency and Severity of Electrical} Shock Injuries

"Electrical shock, electrocution" injuries caused $23 \%$ of all mine electrical injuries and accounted for $26 \%$ of electrical LWDs. Such injuries are often the consequence of working live or in proximity to unguarded live conductors. They account for $63 \%$ of the LWDs attributed to "body systems" and "multiple body parts" from electrical injuries. In addition, $93 \%$ of mine electrical fatalities were attributed to "electrical shock, electrocution". Possible mitigations for electrical shock/ electrocution injuries include the increased use of ground fault circuit interrupters (GFCIs), maintaining the 
proper clearance when working near overhead electric power lines, the use of insulating load link devices and the use of power line proximity and/or contact warning systems.

\section{Mitigating the Frequency and Severity of Electrical Injuries in Maintenance Work Activities}

The WorkActivity "maintenance/repair-electrical” caused $50 \%$ of nonfatal mine electrical accidents and $48 \%$ of the fatalities reported by Work Activity. The Work Activity "maintenance / repair- machinery" caused 13\% of nonfatal mine electrical accidents and $7 \%$ of fatalities. Collectively "maintenance / repair - ...." represents $60 \%$ of all electrical accidents and $53 \%$ of all electrical fatalities. Obviously electrical maintenance is a hazardous WorkActivity deserving special attention.

Possible mitigations for maintenance worker electrical injuries include the increased application of GFCIs and the use of "dead-front" type equipment to isolate maintenance personnel from electrical hazards during troubleshooting. Overall, the safety of electrical maintenance workers could be improved by requiring that each electrical enclosure have a single disconnect mechanismor interlock that de-energized all circuits within an enclosure. This could reduce accidents caused by unintentional contact with adjacent circuits thought to be de-energized or locked-out.

Electrical maintenance/repair workers frequently use meters to troubleshoot live electrical circuits. Examination of accident narratives shows a need for an improved method of accurately verifying meter capabilities and functions in the field to avoid using meters of improper voltage rating or meters set to measure the wrong function.

Suggestions for improving the safe use of electrical meters during live troubleshooting procedures include:

1. color coding or clearly marking meters with their maximum safe voltage and/or current ratings; alternatively, using only single function meters that are color coded or clearly indicate their function(e.g., voltage, current, ohms, etc.). The use of multifunction meters makes it easier for workers to use the wrong meter function or scale;

2 . using meters that autorange up to their maximum voltage and/or current to prevent range selection problems;

3 . using test leads rated for the maximum voltage and/or current of the associated meter, and;

4. using safety test leads with minimal tip exposure to preclude accidental contact with adjacent circuits, not uninsulated or oversized alligator clips.

\section{SUMMARY}

Electrical accidents are the 4th leading cause of death in mining and are disproportionately fatal compared with most other types of mining accidents. It can be argued that every electrical accident is a potential fatality except for some serendipitous set of circumstances that combine to prevent the victim's death.

About one-half of all mine electrical injuries and fatalities occur during electrical maintenance work. Injury severity (average LWDs per LWD accident) increases with age for mining accidents from all causes, but decreases after age 4049 for mine electrical accident victims. Burns are the leading cause of electrical injuries by a nearly 2-to-1 margin, but electrical shock caused $93 \%$ of all mine electrical fatalities. On average, nonfatal electrical shock injuries were more severe (31 LWDs/LWD injury) than nonfatal burn injuries (21 LWDs/LWD injury). Small mines may be more electrically hazardous workplaces than large mines based on total average employment.

Analyzing accident narratives using computerized keyword searches allows rapid identification of core problem areas. Core areas so identified involve "breaker(s)" (313 of 1,926 accidents), "cable(s)" (309 accidents), "batter(y)(ies)" (242 accidents), "energize(d)" (i.e., working live) (163 accidents), "grounds/grounding"(204 accidents), and electrical "meter(s)" (90 accidents).

Improved system design, improved electrical maintenance procedures and schedules, use of power line avoidance devices, power line awareness training, training targeted at known problem areas and vigorous electrical enforcement can combine to improve electrical safety substantially.

\section{REFERENCES}

[1] Title 30, Code of Federal Regulations (CFR) $§ 50.2$, (h)(1-12), available at the website: http://www.msha.gov/regdata/msha/50.2.htm

[2] Bureau of Labor Statistics, U.S. Department of Labor, "Older workers' injuries entail lengthy absences from work”, Summary 96-6, April 1996.

[3] Fotta, Barbara, G. Bockosh, NIOSH Pittsburgh Research Laboratory, CDC, U.S. Department of Health and Human Services, "The aging workforce: an emerging issue in the mining industry", 2000, 13 pp., in press.

[4] Capelli-Schellpfeffer, Mary, Floyd, II, L., Eastwood, K., Liggett, D., "How we can better learn from electrical accidents", IEEE IAS Magazine, May/June 2000, pp. 16-23.

[5] Rossignol, M., M. Pineault, "Classification of fatal occupational electrocutions", Canadian Journal of Public Health., Vol. 85, No. 5, Sept-Oct. 1994, pp. 322-325.

[6] Manuele, Fred A., "Behavioral safety: looking beyond the worker", Occupational Hazards, October 2000, pp. 86-88. 